\title{
Electromagnetic propagation in a turbulent medium: a new approach
}

\author{
Bruno Crosignani \\ Fondazione Ugo Bordoni, Istituto Superiore Poste e Telecomunicazioni, Viale Europa 160, Roma, Italy \\ Paolo Di Porto \\ Istituto di Fisica, Universitò dell'Aquila, L'Aquila, Italy \\ Received January 17, 1983

\begin{abstract}
A formalism based on coupled-mode theory is presented that allows one to deduce the equations of evolution of the correlation functions of the field propagating in a turbulent medium. As a particular application, the second-order correlation function is evaluated under less stringent conditions than those usually required in the frame of optical propagation theories in random media.
\end{abstract}

\section{INTRODUCTION}

Propagation of optical waves in a turbulent medium has been the object of intensive experimental and theoretical investigations during the past 20 years. This had led to the development of different theories of electromagnetic (em) propagation, ${ }^{1-3}$ each one possessing its specific merits and limitations and the last ones being expressed by a number of assumptions underlying the formalism. Among these theories, the moment method, ${ }^{3}$ which directly looks for equations describing the evolution of the correlation functions of the electric field, appears to be powerful and elegant. It permits, in particular, the writing of a parabolic equation for the mutual coherence function, which can be solved in a number of relevant situations. ${ }^{2}$

In the same spirit, we have introduced ${ }^{4}$ a formalism that still leads, as an application, to an evaluation of the mutual coherence function while permitting the release of some of the hypotheses usually employed in the frame-of-the-moment method. Since this last approach seems to be one of the most important new contributions in the theory of wave propagation in a turbulent medium, we consider the development of our alternative method to be of interest. Basically, our method consists of adapting the formalism of coupled-mode theory, usually employed for describing electromagnetic propagation and mode coupling in an imperfect optical fiber, ${ }^{5}$ to the case of propagation in an unbounded random medium. However, the imperfections responsible for mode coupling inside a fiber are of a static nature so there is some difficulty in interpreting ensemble-averaged quantities in terms of real-life measurements; refractive-index variations are time dependent in a turbulent medium, so, actually, mode-coupled theory finds its natural field of application there.

\section{COUPLED-MODE THEORY}

Monochromatic wave propagation in a nonmagnetic medium possessing a refractive index $n(x, y, z)$ is usually dealt with starting from the wave equation, which is a consequence of
Maxwell's equations

$$
\begin{aligned}
& \nabla \times \mathbf{H}=\mathbf{i} \omega \epsilon_{0} n^{2} \mathbf{E}, \\
& \nabla \times \mathbf{E}=-i \omega \mu_{0} \mathbf{H} .
\end{aligned}
$$

In the frame of coupled-mode theory, one can look for solutions of Eqs. (1) and (2) directly without using the wave equation, thus avoiding the introduction of derivatives of an order higher than the first. This is accomplished ${ }^{5}$ by expanding the fields in terms of a suitable complete set of orthogonal functions representing the normal modes associated with a suitably chosen ideal refractive-index distribution $n_{1}(x$, $y$ ) differing from the actual one for the absence of fluctuations.

The description of propagation in an unbounded random medium is achieved by choosing as ideal normal modes the ones pertaining to a homogeneous medium possessing a constant refractive index $n_{1}$, which read

where

$$
\mathbf{E}_{\boldsymbol{\chi}}(\mathbf{r}, z)=(1 / 2 \pi) \hat{e}(\chi) \exp \left(-i \chi \cdot \mathbf{r}-i \beta_{\mathbf{\chi}}\right),
$$

$$
\beta_{x}=\left(k^{2}-\chi^{2}\right)^{1 / 2},
$$

with $k=\omega n_{1} / c$ and $\mathbf{r}=(x, y)$, the unit polarization vector $\mathrm{e}(\chi)$ being orthogonal to the wave vector $\mathbf{k}=\left(\chi, \beta_{\chi}\right)$. We assume that the input field at $z=0$ is polarized along the $x$ axis $\left(E_{y}\right.$ $=0$ ), with a small component $E_{z}$ resulting from an eventual small angular divergence of the beam. As is well known, the smallness of the refractive-index fluctuations forbids the occurrence of appreciable depolarization and backscattering effects when a light beam propagates over considerably long distances in turbulent atmosphere. ${ }^{3}$ This circumstance allows us to simplify the formalism by assuming that the forwardtraveling field is linearly polarized $\left(E_{y}=E_{z}=0\right)$ and by neglecting its coupling with backward-traveling modes. In this way, the electric field reads

$$
E(\mathbf{r}, z, t)=\int \mathrm{d} \chi E_{\boldsymbol{\chi}}(\mathbf{r}, z) e^{i \omega t} c_{\boldsymbol{\chi}}(z),
$$


where the expansion coefficients $c_{\chi}(z)$ obey the set of coupled equations

$$
\mathrm{d} c_{\chi}(z) / \mathrm{d} z=\int \mathrm{d} \chi^{\prime} \exp \left[i\left(\beta_{\chi}-\beta_{\chi^{\prime}}\right) z\right] K_{\chi, \chi^{\prime}}(z) c_{\chi^{\prime}}(z) .
$$

We note that, whereas in general $\chi$ may assume all possible values, our assumption on the input field limits the range of integration over $\chi$ to real values such that

$$
\begin{gathered}
\chi \ll k, \\
\beta_{\chi} \cong k-\chi^{2} / 2 k .
\end{gathered}
$$

The coupling coefficients $K_{\chi, x^{\prime}}(z)$ are furnished by the expression

$$
K_{\mathbf{x}, \mathbf{\chi}^{\prime}}(z)=-i\left(k_{0} / 4 \pi^{2}\right) \int \mathrm{d} \mathbf{r} \delta n(\mathbf{r}, z) \exp \left[i\left(\chi-\chi^{\prime}\right) \cdot \mathbf{r}\right],
$$

where $k_{0}=\omega / c$ and $\delta n(\mathbf{r}, z)$ obey the relation

$$
n(\mathbf{r}, z)=n_{1}+\delta n(\mathbf{r}, z) \text {, }
$$

$n(\mathbf{r}, z)$ being the actual refractive index.

The procedure that, starting from Eqs. (6) and (9), leads to the evaluation of the significant quantities

$$
X_{x, x^{\prime}}(z)=\left\langle c_{\chi}(z) c_{\chi^{\prime}} *(z)\right\rangle,
$$

with the angular brackets indicating an ensemble average over the possible realizations of the system, is now completely analogous to that followed for optical fibers. ${ }^{6}$ Under the hypothesis of slow variation of the $X_{x, x^{\prime}}$ 's over a scale of the order of the typical longitudinal correlation length of the correlation function $\left\langle\delta n(\mathbf{r}, z) \delta n\left(\mathbf{r}, z^{\prime}\right)\right\rangle$, it is possible to obtain a closed system of integrodifferential equations describing the $z$ dependence of $X_{x, x^{\prime}}$ 's induced by the presence of refractive-index fluctuations. More precisely, after assuming that the turbulent medium is homogeneous and isotropic and introducing the correlation function

$$
\left\langle K_{\chi, \chi^{\prime}}(z) K_{\xi, \xi^{\prime}}(0)\right\rangle=\delta^{(2)}\left(\chi-\chi^{\prime}+\xi-\xi^{\prime}\right) F\left(\left|\xi-\xi^{\prime}\right|,|z|\right),
$$

where $\delta^{(2)}$ is the two-dimensional delta function and [see Eq. (9)]

$$
\begin{array}{r}
F\left(\left|\xi-\xi^{\prime}\right|,|z|\right)=-\left(k_{0} / 2 \pi\right)^{2} \int_{-\infty}^{+\infty} \mathrm{d} \mathbf{r}\langle\delta n(\mathbf{r}, z) \delta n(0,0)\rangle \\
\times \exp \left[-i\left(\xi-\xi^{\prime}\right) \cdot \mathbf{r}\right],
\end{array}
$$

one has

$$
\begin{aligned}
\mathrm{d} X_{\chi, x^{\prime}} / \mathrm{d} z= & X_{\chi, \chi^{\prime}} \int \mathrm{d} \chi^{\prime \prime}\left[g\left(\chi^{\prime \prime}, \chi\right)+g\left(\chi^{\prime}, \chi^{\prime \prime}\right)\right] \\
& -\iint \mathrm{d} \chi^{\prime \prime} \mathrm{d} \chi^{\prime \prime \prime} \exp \left[i \left(\beta_{\chi}-\beta_{\chi^{\prime \prime}}\right.\right. \\
& \left.\left.+\beta_{\chi^{\prime \prime \prime}}-\beta_{\chi^{\prime}}\right) z\right] \delta^{(2)} \\
& \times\left(\chi-\chi^{\prime \prime}+\chi^{\prime \prime \prime}-\chi^{\prime}\right) g\left(\chi^{\prime \prime \prime}, \chi^{\prime}\right) X_{\chi^{\prime \prime}, \chi^{\prime \prime}} \\
& -\iint \mathrm{d} \chi^{\prime \prime} \mathrm{d} \chi^{\prime \prime \prime} \exp \left[i \left(\beta_{\chi}-\beta_{\chi^{\prime}}\right.\right. \\
& \left.\left.+\beta_{\chi^{\prime \prime}}-\beta_{\chi^{\prime \prime \prime}}\right) z\right] \delta^{(2)} \\
& \times\left(\chi^{\prime \prime}-\chi^{\prime}+\chi-\chi^{\prime \prime \prime}\right) g\left(\chi, \chi^{\prime \prime \prime}\right) X_{\chi^{\prime \prime}, \chi^{\prime \prime}}
\end{aligned}
$$

with

$$
g\left(\chi^{\prime \prime}, \chi\right)=\int_{0}^{+\infty} \mathrm{d} z F\left(\left|\chi^{\prime \prime}-\chi\right|, z\right) \exp \left[-i\left(\beta_{\chi^{\prime \prime}}-\beta_{\chi}\right) z\right] .
$$

In particular, the diagonal elements are mutually coupled through the set of equations

$$
\mathrm{d} X_{\chi, \chi} / \mathrm{d} z=X_{\chi, \chi} \int \mathrm{d} \chi^{\prime} h\left(\chi, \chi^{\prime}\right)-\int \mathrm{d} \chi^{\prime} h\left(\chi, \chi^{\prime}\right) X_{\chi^{\prime}, \chi^{\prime}}
$$

where

$$
\begin{aligned}
& h\left(\chi^{\prime \prime}, \chi\right)=2 \operatorname{Re}\left[g\left(\chi^{\prime \prime}, \chi\right)\right] \\
& \quad=\int_{-\infty}^{+\infty} \mathrm{d} z F\left(\left|\chi^{\prime \prime}-\chi,\right| z \mid\right) \exp \left[-i\left(\beta_{\chi^{\prime \prime}}-\beta_{\chi}\right) z\right]
\end{aligned}
$$

is a negative quantity, from which energy conservation can immediately be deduced in the form

$$
(\mathrm{d} / \mathrm{d} z) \int \mathrm{d} \chi X_{x, x}=0 .
$$

In principle, the set of Eq. (14) can be solved once all the


3 , it is possible under certain assumptions to transform Eq. (14) into a set of uncoupled diffusion equations (in $\chi$ space), which can easily be integrated.

\section{DERIVATION OF THE DIFFUSION EQUATION}

The expressions of $g\left(\chi^{\prime \prime}, \chi\right)$ and $h\left(\chi^{\prime \prime}, \chi\right)$ can be put in a simple form if we observe that [see Eq. (8)]

$$
\beta_{\chi^{\prime \prime}}-\beta_{\chi} \cong(1 / 2 k)\left(\chi^{2}-\chi^{\prime \prime}{ }^{2}\right)=(1 / 2 k)\left(\chi+\chi^{\prime \prime}\right)\left(\chi-\chi^{\prime \prime}\right)
$$

so that the exponential appearing in Eqs. (15) and (17) can be approximated by unity as long as

$$
(1 / 2 k)\left(\chi+\chi^{\prime \prime}\right)\left|\chi-\chi^{\prime \prime}\right| z \ll 1 .
$$

In turn, expression (20) is equivalent to the condition

$$
\Delta \chi / k \ll 1
$$

( $\Delta \chi$ denoting the relevant range of values of $\chi$ ), which follows from the fact that $F\left(\left|\chi^{\prime \prime}-\chi\right|,|z|\right)$ becomes negligible for $\mid \chi^{\prime \prime}$ $-\chi \mid>1 / d$ and $|z|>d, d$ representing the typical correlation length of the refractive-index fluctuations. Since expression (21) is in practice satisfied for any $z$ under our assumption of small angular divergence of the beam, we can write

$$
\begin{aligned}
g\left(\chi^{\prime \prime}, \chi\right) & =\frac{1}{2} \int_{-\infty}^{+\infty} \mathrm{d} z F\left(\left|\chi^{\prime \prime}-\chi\right|,|z|\right) \\
& =g\left(\left|\chi^{\prime \prime}-\chi\right|\right)=\frac{1}{2} h\left(\left|\chi^{\prime \prime}-\chi\right|\right) .
\end{aligned}
$$

An argument analogous to that given above allows us to set equal to unity the exponential appearing on the right-hand side of Eq. (14). More precisely, if we consider, for example the second term, we can write, as an approximation,

$$
\left(\beta_{\chi}-\beta_{\chi^{\prime \prime}}+\beta_{\chi^{\prime \prime \prime}}-\beta_{\chi^{\prime}}\right)=(1 / 2 k)\left(\chi^{\prime \prime 2}-\chi^{2}+\chi^{\prime 2}-\chi^{\prime \prime \prime}{ }^{2}\right) \text {, }
$$


so that $\left|\beta_{\chi}-\beta_{\chi^{\prime \prime}}+\beta_{\chi^{\prime \prime \prime}}-\beta_{\chi^{\prime}}\right|$ turns out to be less than the smallest of the two quantities

$$
(1 / 2 k)\left|\chi^{\prime \prime}-\chi^{\prime \prime \prime}\right|\left(\chi+\chi^{\prime}+\chi^{\prime \prime}+\chi^{\prime \prime \prime}\right)
$$

and

$$
(1 / 2 k)\left|\chi^{\prime}-\chi^{\prime \prime \prime}\right|\left(\chi+\chi^{\prime}+\chi^{\prime \prime}+\chi^{\prime \prime \prime}\right),
$$

as can easily be verified by using the relation $\left|\chi^{\prime}-\chi\right| \leqq \mid \chi^{\prime}$ $-\chi \mid$ and taking into account the constraint imposed by the delta function appearing in the integrand. Accordingly, the exponential can be approximated by unity as long as

$$
\frac{z}{k} \frac{\Delta \chi}{l} \ll 1
$$

where $l$ is given, respectively, by $d$ or by the inverse of the interval $\left|\chi^{\prime \prime}-\chi^{\prime \prime \prime}\right|$ over which $X_{\chi^{\prime \prime}, \chi^{\prime \prime \prime}}$ becomes negligible, according to whether expression (25) or (24) applies. A similar argument can be employed for the third term on the right-hand side of Eq. (14).

Expressions (21) and (26) both have to be checked $a$ posteriori once the solutions to Eqs. (14) and (16) have been found by assuming their validity. If expression (26) holds true, then Eq. (14) can be rewritten in the form

$$
\begin{aligned}
\mathrm{d} X_{\chi, \chi^{\prime}} / \mathrm{d} z= & X_{\chi, \chi^{\prime}} \int \mathrm{d} \chi^{\prime \prime}\left[g\left(\chi^{\prime \prime}, \chi^{\prime}\right)+g\left(\chi^{\prime}, \chi^{\prime \prime}\right)\right] \\
& -\int \mathrm{d} \chi^{\prime \prime} g\left(\chi^{\prime \prime}, \chi^{\prime}\right) X_{\chi^{\prime \prime}+\chi-\chi^{\prime}, \chi^{\prime \prime}} \\
& -\int \mathrm{d} \chi^{\prime \prime} g\left(\chi, \chi^{\prime \prime}\right) X_{\chi^{\prime \prime}, \chi^{\prime \prime}+\chi^{\prime}-\chi},
\end{aligned}
$$

which, if one takes advantage of Eq. (22) for extending without sensible error the range of the integrals appearing in Eq. (27) to the whole $\chi$ space, reduces to

$$
\mathrm{d} X_{\chi, \chi^{\prime}} / \mathrm{d} z=2 X_{\boldsymbol{x}, \boldsymbol{x}^{\prime}} \int \mathrm{d} \xi g(\xi)-2 \int \mathrm{d} \xi g(\xi) X_{\chi+\xi, \chi^{\prime}+\xi}
$$

If we now observe that the second term on the right-hand side of Eq. (28) is responsible for the coupling of the $X_{x, x^{\prime}}$ to the terms $X_{\chi^{\prime \prime \prime}, \chi^{\prime \prime}}$ such that $\chi^{\prime \prime \prime}-\chi^{\prime \prime}=\chi-\chi^{\prime}$ [the origin of this behavior being traceable to the delta function appearing in Eq. (14)], one can regard Eq. (28) as an equation in the variable $\chi$ for every fixed value of $\eta=\chi^{\prime}-\chi$ and solve accordingly in terms of the boundary condition $X_{\chi, \chi+\eta}(z=0)$. After this, one can finally evaluate the autocorrelation function of the electric field through the relation

$$
\begin{aligned}
& \left\langle E(\mathbf{r}, z, t) \mathbf{E}^{*}\left(\mathbf{r}^{\prime}, z, t\right)\right\rangle=\Gamma\left(\mathbf{r}, \mathbf{r}^{\prime}, z\right)=\left(1 / 4 \pi^{2}\right) \iint \mathrm{d} \chi \mathrm{d} \eta \\
& \quad \times \exp \left[-i \chi \cdot\left(\mathbf{r}-\mathbf{r}^{\prime}\right)+i \eta \cdot \mathbf{r}^{\prime}+i \beta_{\mid \eta+\chi} z-i \beta_{\chi} z\right]_{X_{\mathbf{x}, \boldsymbol{\eta}+\boldsymbol{\eta}}(z)}
\end{aligned}
$$

Following this procedure, let us expand $X_{\chi+\xi, \chi^{\prime}+\xi}$ around $\xi=$ 0 , thus getting

$$
\begin{aligned}
X_{\chi+\xi, \chi^{\prime}+\xi}= & X_{\chi, x^{\prime}}+\left(\partial X_{\chi, x^{\prime}} / \partial \chi\right) \cdot \xi \\
& +\frac{1}{2}\left(\partial^{2} X_{\chi, x^{\prime}} / \partial \chi \partial \chi\right): \xi \xi+\ldots,
\end{aligned}
$$

which, once inserted into Eq. (28), yields the diffusion equation

$$
\partial X_{\chi, \chi+\eta} / \partial z-D\left(\partial^{2} / \partial \chi_{x}^{2}+\partial^{2} / \partial \chi_{y}^{2}\right) X_{\chi, \chi+\eta}=0,
$$

where

$$
D=-(1 / 2) \int \mathrm{d} \xi \xi^{2} g(\xi)
$$

In deriving Eq. (31), we have omitted all terms containing odd-order derivatives, since their coefficients are obviously zero because of the homogeneity and isotropy of the medium, and we have neglected even terms of order higher than the second in the spirit of diffusionlike theory.

If, in particular, we assume a Gaussian behavior of the refractive-index fluctuations, that is,

$\left\langle\delta n(\mathbf{r}, z) \delta n\left(\mathbf{r}^{\prime}, z^{\prime}\right)\right\rangle=\left\langle\delta n^{2}\right\rangle \exp \left\{\left[-\left|\mathbf{r}-\mathbf{r}^{\prime}\right|^{2}-\left(z-z^{\prime}\right)^{2}\right] / d^{2}\right\}$,

we obtain, through Eqs. (13), (17), (22), (32), and (33), the following expression for the diffusion coefficient $D$ :

$$
D=\sqrt{\pi} k^{2}\left\langle\delta n^{2}\right\rangle / d .
$$

\section{EVALUATION OF THE MUTUAL COHERENCE FUNCTION}

In this section we explicitly evaluate the mutual coherence function $\Gamma\left(\mathbf{r}, \mathbf{r}^{\prime}, z\right)$ defined in Eq. (29) for two significant input fields.

\section{Constant-Amplitude Wave}

The field $E(\mathbf{r}, z=0)$ is assumed to be of the form

$$
E(\mathbf{r}, z=0)=E_{0} \exp [i \phi(\mathbf{r})],
$$

where the phase $\phi(\mathbf{r})$ is a fluctuating quantity, which corresponds, for example, to an ideal plane wave after crossing a thin screen. ${ }^{2}$ Furthermore, we assume an initially Gaussian mutual coherence function,

$$
\left\langle\exp \left[i \phi(\mathbf{r})-i \phi\left(\mathbf{r}^{\prime}\right)\right]\right\rangle=\exp \left[-\left|\mathbf{r}-\mathbf{r}^{\prime}\right|^{2} / b^{2}\right],
$$

so that the ideal plane-wave case is simply obtained by letting $b$ go to infinity.

According to Eqs. (3), (5), and (35), we can write

$$
c_{\boldsymbol{\chi}}(0)=\left(E_{0} / 2 \pi\right) \int \mathrm{d} \mathbf{r} \exp [i \chi \cdot \mathbf{r}+i \phi(\mathbf{r})],
$$

which yields, with the help of Eq. (36),

$$
X_{\boldsymbol{x}, \boldsymbol{\chi}+\boldsymbol{\eta}}(0)=\left|E_{0}\right|^{2} \delta^{2}(\eta) b^{2} \exp \left(-\chi^{2} b^{2} / 4\right) .
$$

On the other hand, the solution of Eq. (31) can be written in the standard form

$X_{\chi, \chi+\eta}(z)=\int_{-\infty}^{+\infty} \mathrm{d} \beta Y_{\eta}(\beta) \exp \left(-D \beta^{2} z+i \beta \cdot \chi\right)$,

where

$$
Y_{\eta}(\beta)=\left(1 / 4 \pi^{2}\right) \int \mathrm{d} \chi \exp (-i \beta \cdot \chi) X_{\chi, \chi+\eta}(0) .
$$

By inserting Eq. (38) into Eq. (40), we obtain

$$
Y_{\eta}(\beta)=\left|E_{0}\right|^{2} \delta^{(2)}(\eta) \exp \left(-\beta^{2} / b^{2}\right),
$$


which, once inserted into Eq. (39), furnishes

$$
X_{\chi, \chi+\eta}(z)=\left|E_{0}\right|^{2} \delta^{(2)}(\eta) \pi A \exp \left(-A \chi^{2} / 4\right),
$$

where

$$
1 / A=D z+1 / b^{2} .
$$

By means of Eqs. (29) and (42), it is finally possible to evaluate the mutual coherence function of the electric field, which reads

$$
\Gamma\left(\mathbf{r}, \mathbf{r}^{\prime}, z\right)=\left|E_{0}\right|^{2} \exp \left(-\left|\mathbf{r}-\mathbf{r}^{\prime}\right|^{2} / A\right) .
$$

In particular, if $b \rightarrow \infty$, we have

$$
\Gamma\left(\mathbf{r}, \mathbf{r}^{\prime}, z\right)=\left|E_{0}\right|^{2} \exp \left(-\left|\mathbf{r}-\mathbf{r}^{\prime}\right|^{2} D z\right),
$$

which describes the ideal plane-wave case.

\section{Beam Wave}

The field at $z=0$ is a deterministic quantity of the form

$$
E(\mathbf{r}, z=0)=E_{0} \exp \left(-2 r^{2} / b^{2}\right),
$$

so that

$$
c_{\chi}(0)=\left(E_{0} b^{2} / 4\right) \exp \left(-\chi^{2} b^{2} / 8\right)
$$

and

$$
X_{x, x+\eta}(0)=\left(\left|E_{0}\right|^{2} b^{4} / 16\right) \exp \left(-\chi^{2} b^{2} / 4-\eta^{2} b^{2} / 8-\eta \cdot \chi b^{2 / 4}\right),
$$

which yields, with the help of Eqs. (39) and (40),

$$
X_{x, \boldsymbol{\chi}+\eta}(z)=\left|E_{0}\right|^{2}(b / 4)^{2} A \exp \left[-b^{2} \eta^{2} / 4-\right]|(\chi+\eta / 2)|^{2} A / 4 .
$$

We can now insert Eq. (49) into Eq. (29), which, after we take advantage of expression (19) to write

$$
\exp \left[i\left(\beta_{|x+\eta|}-\beta_{\chi}\right) z\right]=\exp \left[-i\left(\eta^{2}+2 \eta \cdot \chi\right)(z / 2 k)\right]
$$

and after a rather lengthy if otherwise straightforward calculation, gives

$$
\begin{array}{r}
\Gamma\left(\mathbf{r}, \mathbf{r}^{\prime}, z\right)=\left[\left|E_{0}\right|^{2} /\left(1+Z^{2} / A\right)\right] \exp \left\{-\left[\left|\mathbf{r}-\mathbf{r}^{\prime}\right|^{2} / A+\left|\mathbf{r}+\mathbf{r}^{\prime}\right|^{2} / b^{2}\right.\right. \\
\left.\left.+2 i Z\left(r^{2}-r^{\prime 2}\right) / b A\right] /\left(1+Z^{2} / A\right)\right\}, \quad(51)
\end{array}
$$

where $Z=4 z / b k$, which coincides with Eq. (45) in the limit $b \rightarrow \infty$, as it must.

\section{CONCLUSIONS}

We have developed a new approach to the problem of electromagnetic propagation in a turbulent medium based on coupled-mode theory that permits, in particular, the evaluation of the mutual coherence function and can thus be directly compared with the moment method in which the equations for the moments of the field are derived. ${ }^{1}$ To this end, we recall that the moment approach, as well as the other methods employed for describing electromagnetic propagation in a turbulent atmosphere, is based on four critical assumptions, which consist of ${ }^{3}$ (1) neglecting depolarization effects, (2) neglecting backscattering, (3) approximating the full wave equation for the field in the parabolic form, and (4) approximating the correlation function of the refractive-index fluctuations with a delta function for what concerns its $z$ dependence.

Our method indeed includes the first three assumptions [the third one corresponding to expression (8)], which are of a basic nature and turn out to be verified, under normal conditions, over long traveled paths, but it does allow us to discard the fourth (rather qualitative) condition. More precisely, the last assumption is, for all practical purposes, replaced by the hypothesis underlying coupled-mode theory of slow variation of the $X_{x, x^{\prime}}(z)$ 's over a scale of the order of $d$ (see Section 2).

In order to set expressions (21) and (26) in explicit form, we have to evaluate $\Delta \chi$ and $l$ in a self-consistent.way by means of the solutions found in Section 4. In the constant-amplitude case, we can immediately infer from Eq. (42) that $l=\infty$ and $\Delta \chi \cong A^{-1 / 2}$, so expression (26) is always satisfied, while expression (21) becomes [for $D z \gg 1 / b^{2}$, see Eq. (43)]

$$
1 /\left(k A^{1 / 2}\right)=(D z)^{1 / 2} / k=\pi^{1 / 4}\left\langle\delta n^{2}\right\rangle^{1 / 2}(z / d)^{1 / 2} \ll 1,
$$

which is in practice fulfilled for any reasonable value of $z$.

In the beam-wave case, one still has, according to Eq. (49), $\Delta \chi \cong A^{-1 / 2}$ and, correspondingly, Eq. (52), but now $l$ is finite and of the order of $b$, so that the limitation expressed by expression (26) becomes effective and yields

$$
(D z)^{1 / 2} z /(b k)=\pi^{1 / 4}\left\langle\delta n^{2}\right\rangle^{1 / 2}(z / d)^{1 / 2}(z / b) \ll 1 .
$$

\section{ACKNOWLEDGMENTS}

We wish to thank M. Carnevale for many helpful discussions. This work was partially supported by the Italian National Research Council (CNR).

\section{REFERENCES}

1. V. I. Tatarskii, The Effects of the Turbulent Atmosphere on Wave Propagation (National Technical Information Service, Springfield, Va., 1971).

2. A. Ishimaru, Wave Propagation and Scattering in Random Media (Academic, New York, 1978).

3. J. W. Strohbehn, "Modern theories in the propagation of optical waves in a turbulent medium,' in Laser Beam Propagation in the Atmosphere, J. W. Strohbehn, ed. (Springer-Verlag, Berlin, 1978).

4. For a preliminary description of this procedure, see M. Carnevale, B. Crosignani, and P. Di Porto, "Coupled-mode theory approach to electromagnetic propagation through a random medium," Phys. Rev. Lett. 49, 916-918 (1982).

5. D. Marcuse, Theory of Dielectric Optical Waveguides (Academic, New York, 1974).

6. B. Crosignani, B. Daino, and P. Di Porto, "Statistical coupled equations in lossless optical fibers," IEEE Trans. Microwave Theory Tech. MTT-23, 416-420 (1975). 